\title{
金属离子水解时多核络合形成 曲线的线性特性
}

\author{
杜有明梁家昌 \\ （天津广播电视大学）（中国民用航空学院）
}

虽然有了一些分析与计算多核络合物稳定常数的方法 ${ }^{[1-6]}$, 但研究溶液中多核络 合物 的 形成及其稳定性仍是一个复杂的问题. 在本工作中我们将考察金属离子水解时的多核络合形 成曲线的线性特性,它也是与溶液中实际形成的多核络 合体系的稳定性密切相关的.

设在溶液中存在配位体 $A$ 与金属离子 $B$, 它们形成单核与多核络合物 $A_{p} B_{q}$ (为书写方便 起见, 已将电荷符号略去). 为了维持其活度系数不变, 络合应在恒定的离子强度溶液中进行. 这时质量平衡方程可表为

$$
\begin{aligned}
& A=a+\sum_{p} \sum_{q} p\left[A_{p} B_{q}\right], \\
& B=b+\sum_{p} \sum_{q} q\left[A_{p} B_{q}\right],
\end{aligned}
$$

其中 $A$ 与 $B$ 分别为配位体与金属离子总浓度, $a$ 与 $b$ 分别为它们的自由浓度, $\left[A_{p} B_{q}\right]$ 为络合 物 $A_{p} B_{q}$ 的平衡浓度, 求和号中的 $p$ 与 $q$ 只取与溶液中所实际存在的那些络合物相对应的值.

引人络合物 $A_{p} B_{q}$ 的稳定常数 $X_{p q}$ 、络合形成函数 $\bar{n}$ 及络合度 $Y$, 它们分别等于

$$
\begin{aligned}
X_{p q} & =\frac{\left[A_{p} B_{q}\right]}{a^{p} b^{q}}, \\
\bar{n} & =\frac{A-a}{B}, \\
Y & =\frac{B}{b} .
\end{aligned}
$$

由方程(1)-(5)可得

$$
\begin{aligned}
& \bar{n}=\frac{\sum_{p} \sum_{q} p a^{p} b^{q-1} X_{p q}}{1+\sum_{p} \sum_{q} q a^{p} b^{q-1} X_{p q}}, \\
& Y-1+\sum_{p} \sum_{q} q a^{p} b^{q-1} X_{p q q} .
\end{aligned}
$$

由方程 (1)-(7)可推得络合形成函数 $\bar{n}$ 与络合度 $Y$ 之间的关系式. Hedstrom ${ }^{[7]}$ 首先作了 这方面的工作,但更为实用的关系式 ${ }^{[3]}$ 则是

$$
\ln Y=\int_{\ln a_{1}}^{\ln a}\left[\bar{n}+\left(\frac{\partial \bar{n}}{\partial \ln B}\right)_{a}\right]_{B} d \ln a,
$$

本文1981年11月17日收到. 1982 年 6 月 3 日收到改稿. 
其中 $a_{1}$ 乃是配位体自由浓度 $a$ 的初值. 可见 $\bar{n}$ 与 $Y$ 不是互为独立的函数.

实验表明,金属离子水解时，常常生成多核络合体系. 对此体系不难测得其实验曲线筷 $\bar{n}(\log a)_{\mathbf{B}}$ ，它代表在一系列不同的金属离子初始浓度下，络合形成函数随配位体自由浓度对数

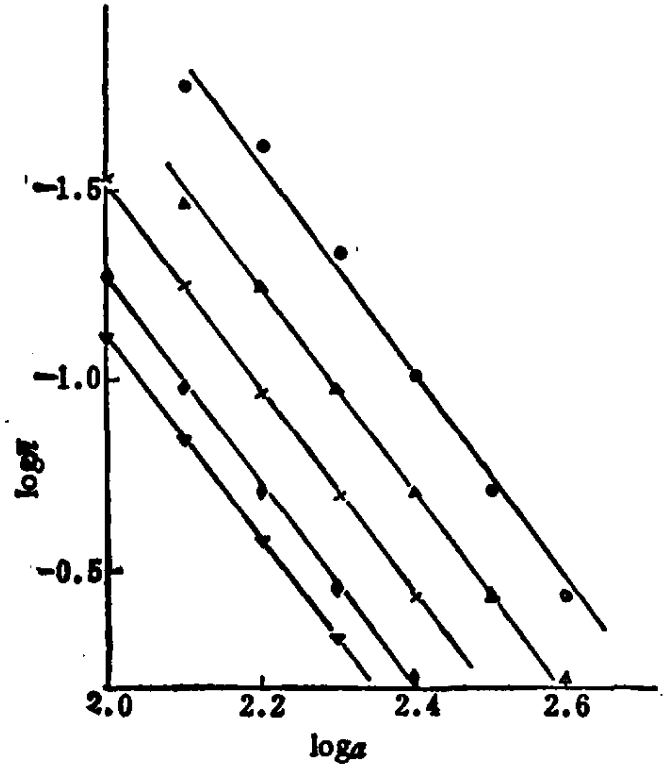

因 $1 \mathrm{Sn}(11)$ 水解时的 $\log \bar{n}(\log a)_{\operatorname{los} b}$

$\odot \log b=-2.3 ; \triangle \log b=-2.1$;

$+\log b=-1.9 ; \diamond \log b=-1.7$

$\nabla \log b=-1.6 ;\left(\frac{\partial \log b}{\partial \log a}\right)_{1}=-2.0$

变化的曲线筷。利用方程式 (5) 与 (8), 不难把实 验曲线筷 $\bar{n}(\log a)_{B}$ 转换成 $\log \bar{n}(\log a)_{b_{i}}(i-1$, $2, \cdots, I)^{[3]}$ 。有意义的是，金属离子水解数据所 给出的 $\log \bar{n}(\log a)_{b i}$ 系互相平行的直线筷, 而 $\log \bar{n}$ $(\log a)_{B}$ 则否. $\mathrm{Sn}(11)$ 水解 ${ }^{(3,0)}$ 时的直线簇 $\log \bar{n}$ $(\log a)_{b_{i}}(i-1,2, \cdots, 5)$ 乃是其典型例子（见图 1).

由此即可推得

或

$$
\left.\begin{array}{c}
\log \vec{n}-\log k_{1}\left(b_{i}\right)+k_{2} \log a \\
\bar{n}-k_{1}\left(b_{i}\right) a^{k_{2}}
\end{array}\right\},
$$

其中在一定温度下 $k_{1}\left(b_{i}\right)$ 是 $b_{i}$ 的函数, $k_{2}$ 为常数.

由方程 $(6)$ 及 $(9)$ 即可获得 $k_{2}(b)$ 与 $X_{p q}$ 间的关 系式为

$$
\begin{aligned}
& k_{1}(b)-\lim _{\log a \rightarrow 0} \bar{n} \\
& -\frac{\sum_{p} \sum_{q} p b^{q-1} X_{p q}}{1+\sum_{p} \sum_{q} q b^{q-1} X_{p_{q}}},
\end{aligned}
$$

(10)式表明了 $k_{1}(b)$ 值与络合物 $A_{p} B_{q}$ 的平衡常数 $X_{p q}$ 直接相关.

这样, 利用直线筷 $\log \bar{n}(\log a)_{b_{i}}(i-1,2, \cdots, I)$ 就可由图解法获得 $k_{1}\left(b_{i}\right)$ 之值，再利用 方程 $(10)$, 可求得少于或等于 $I$ 个的 $X_{p q}$ 值.

当只生成单核络合物 $A_{p} B(p-1,2, \cdots)$ 时, 则 $q=1,(10)$ 式就简化成

$$
k_{1}=\frac{\sum_{p} p X_{p_{1}}}{1+\sum_{p} X_{p_{1}}},
$$

这时 $k_{1}$ 就与 $b$ 值无关了,因而(9)式化为

$$
\bar{n}-k_{1} a^{k_{2}},
$$

(12)式表明,在这种情况下直线筷 $\log \bar{n}(\log a)_{b_{i}}(i-1,2, \cdots, l)$ 就重合成一条直线.

方程(9)一(12)同样适用于用 “自介质” 法所获得的金属离子水解时所生成的多核络合体 系. 由于金属离子 $B$ 具有非常高的浓度, 所以它的绝大部分均以自由离子的形式存在于溶 液中, 只有极少部分与配位体 $A$ 络合, 因而当 $B$ 足够大、且络合形成函数 $\bar{n}$ 非常小时, 在测量 误差范围内可取 $b \approx B$. 这样, 反应前后的离子强度仍可视为不变, 上述的方程式仍成 立. $\mathrm{La}(11)$ 水解数据 ${ }^{[a, 9}$ 是在这种实验条件下获得的, 它的 $\log \bar{n}(\log a)_{b_{i}}(i-1,2,3,4)$ 直线 筷见图 2.

表 1 中列出了由图 2 上的直线簇的截距及科率所给出的在四个 $b$ 值下的 $k_{1}(b)$ 与 $k_{2}$ 值. 图 2 显示,当 $\log \bar{n}>(-2.2)$ 时, 实验点就与直线簇 $\log \bar{n}(\log a)_{b_{i}}$ 产生系统偏差, 这并不 
表示方程(9)不适用于“自介质”法, 而只是表明, 随着 $\bar{n}$ 的增加, 在测量误差范围内 $b$ 就不再等 于 $B$ 了，下面将通过平衔常数的计算再来证 实这一点. 在文献 [3] 中已给出了 $\mathrm{La}(11)$ 水解时的主要产物为 $\mathrm{LaOH}^{2+}$ 及 $\mathrm{La}_{2} \mathrm{OH}^{5+}$, 它们的稳定常数分别为

$$
\begin{aligned}
& X_{11}=(7.681 \pm 0.033) \times 10^{-11} M, \\
& X_{12}-(1.06 \pm 0.16) \times 10^{-10} .
\end{aligned}
$$

现在再用方程 (10)及表 1 中的 $k_{1}(b)$ 值来计 算 $X_{11}$ 及 $X_{12}$ 值, 由此得 $X_{11}=7.81 \times 10^{-11} M$, $X_{12}=1.00 \times 10^{-10}$, 两者结果很好相符. 这 也表明了方程(9)一-(12)

同样可用来处理“自介质”法所获得的金属离 子水解数据.

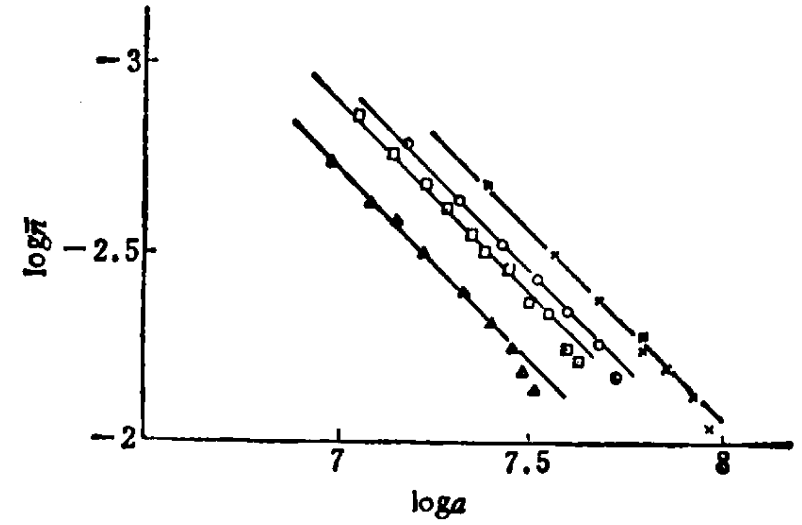

图 2 La(11) 水解时的 $\log \pi(\log a) b_{i}$ 当 $\log \bar{n}<(-2.2)$ 时, $b \approx B$.

$\triangle B=0.967 M ; \quad$ व $B=0.487 M$;

○ $B=0.300 M ; \quad+B=0.097 M$

表 $1 \mathrm{La}(11)$ 水解时的 $k_{1}(b)$ 与 $k_{2}$ 值

\begin{tabular}{c|c|c}
\hline$b$ & $k_{1}(b)$ & $k_{2}$ \\
\hline $0.967 M$ & $1.90 \times 10^{-10}$ & 1.0 \\
$0.487 M$ & $1.29 \times 10^{-10}$ & 1.0 \\
$0.300 M$ & $1.15 \times 10^{-10}$ & 1.0 \\
$0.097 M$ & $8.80 \times 10^{-11}$ & 1.0 \\
\hline
\end{tabular}

不难看出,方程 (12)与 (9) 所给出的曲线簇与低平衡压范围内的异相表面上的 Freundlich 吸附等温线十分相似. 情况确实如此,这只要把金属离子视为吸附剂的吸附中心,把配位体视 为溶质,则溶液中的络合作用就与异相表面上的吸附作用变得相似了 ${ }^{[10]}$. 这正是可用方程(9) 与(12)来表征金属离子水解时络合形成曲线的线性特性的原因. 体系的络合形成及其络合形 成常数随温度的变化系通过 $\log \bar{n}(\log a)_{b_{i}}$ 的平行直线簇的截距和斜率的改变而体现出来的.

\section{结 论}

（1）表明了金属离子水解时其多核络合形成曲线 $\log \vec{n}(\log a)_{b}$ 具有线性规律.

(2) 给出了络合形成函数与络合度间的关系式.

致谢：本工作曾得到过徐光宪老师与陈荣梯老师的关心与指正,谨致谢意.

\section{考文献}

[1] Sillén, L. G., Acta Chem. Scand., 8(1954), 299; ibid., 8(1954), 318.

[2] 徐光宪、橿曾振、严继民, 化学学报, 5 (1959), 229 .

[ 3 ] Лян Цзя-чан (架家昌), Ду Ю-мин (杜有明), Жур. Неоре. Хим., 9 (1964), 1333.

[4] Sillén, L. G., Pure Appl. Chem., 17(1968), 55.

[5] Baes, C. F. \& Mesmer, R. E., The Hydrolysis of Cations, Wiley, New York, 1976.

[6] Rossoti, H., The Study of Ionic Equilibria, Longman, New York, 1978.

[ 7 ] Hedström, B., Acta Chem. Scand., 9(1955), 613.

[8] Tobias, R. 8., Acta Chem. Scand., 12(1958), 198.

[9] Biedermann, G. \& Ciavatta, L., Acta Chem. Scand., 15(1961), 1347.

[10] 徐光宪, 化学学报, 27 (1961), 93.

第 13 期 